\title{
Video Article \\ Production of Pseudotyped Particles to Study Highly Pathogenic Coronaviruses in a Biosafety Level 2 Setting
}

\author{
Jean K. Millet ${ }^{1,2}$, Tiffany Tang ${ }^{3}$, Lakshmi Nathan ${ }^{3}$, Javier A. Jaimes ${ }^{4}$, Hung-Lun Hsu ${ }^{3,5}$, Susan Daniel ${ }^{3}$, Gary R. Whittaker ${ }^{1}$ \\ ${ }^{1}$ Department of Microbiology and Immunology, College of Veterinary Medicine, Cornell University \\ ${ }^{2}$ INRA, Virologie et Immunologie Moléculaires \\ ${ }^{3}$ Robert Frederick Smith School of Chemical and Biomolecular Engineering, Cornell University \\ ${ }^{4}$ Department of Microbiology, College of Agricultural and Life Sciences, Cornell University \\ ${ }^{5}$ Horae Gene Therapy Center, University of Massachusetts Medical School
}

Correspondence to: Gary R. Whittaker at gary.whittaker@cornell.edu

URL: https://www.jove.com/video/59010

DOI: doi:10.3791/59010

Keywords: Cancer Research, Issue 145, Pseudotyped particle, pseudovirion, coronavirus, CoV, spike protein, severe acute respiratory syndrome coronavirus, SARS-CoV, Middle East respiratory syndrome coronavirus, MERS-CoV, murine leukemia virus, MLV

Date Published: 3/1/2019

Citation: Millet, J.K., Tang, T., Nathan, L., Jaimes, J.A., Hsu, H.L., Daniel, S., Whittaker, G.R. Production of Pseudotyped Particles to Study Highly Pathogenic Coronaviruses in a Biosafety Level 2 Setting. J. Vis. Exp. (145), e59010, doi:10.3791/59010 (2019).

\section{Abstract}

The protocol aims to generate coronavirus (CoV) spike (S) fusion protein pseudotyped particles with a murine leukemia virus (MLV) core and luciferase reporter, using a simple transfection procedure of the widely available HEK-293T cell line. Once formed and released from producer cells, these pseudovirions incorporate a luciferase reporter gene. Since they only contain the heterologous coronavirus spike protein on their surface, the particles behave like their native coronavirus counterparts for entry steps. As such, they are the excellent surrogates of native virions for studying viral entry into host cells. Upon successful entry and infection into target cells, the luciferase reporter gets integrated into the host cell genome and is expressed. Using a simple luciferase assay, transduced cells can be easily quantified. An important advantage of the procedure is that it can be performed in biosafety level 2 (BSL-2) facilities instead of BSL-3 facilities required for work with highly pathogenic coronaviruses such as Middle East respiratory syndrome coronavirus (MERS-CoV) and severe acute respiratory syndrome coronavirus (SARS$\mathrm{CoV}$ ). Another benefit comes from its versatility as it can be applied to envelope proteins belonging to all three classes of viral fusion proteins, such as the class I influenza hemagglutinin (HA) and Ebola virus glycoprotein (GP), the class II Semliki forest virus E1 protein, or the class III vesicular stomatitis virus $\mathrm{G}$ glycoprotein. A limitation of the methodology is that it can only recapitulate virus entry steps mediated by the envelope protein being investigated. For studying other viral life cycle steps, other methods are required. Examples of the many applications these pseudotype particles can be used in include investigation of host cell susceptibility and tropism and testing the effects of virus entry inhibitors to dissect viral entry pathways used.

\section{Video Link}

The video component of this article can be found at https://www.jove.com/video/59010/

\section{Introduction}

Host cell entry constitutes the initial steps of the viral infectious life cycle. For enveloped viruses, this involves binding to a single host cell receptor or several receptors, followed by fusion of viral and cellular membranes. These essential functions are carried out by viral envelope glycoproteins $s^{1,2}$. The coronavirus envelope glycoprotein is called the spike (S) protein and is a member of the class I viral fusion proteins ${ }^{2,3,4,5,6}$ Studying viral envelope glycoproteins is critical for understanding many important characteristics of a given virus, such as: lifecycle initiation, its host and cellular tropism, interspecies transmission, viral pathogenesis, as well as host cell entry pathways. Viral pseudotyped particles, also named pseudovirions, are powerful tools that enable us to easily study the function of viral fusion proteins. Pseudotyped particles or pseudovirions are chimeric virions that consist of a surrogate viral core with a heterologous viral envelope protein at their surface. The protocol's main purpose is to show how to obtain coronavirus spike pseudotyped particles that are based on a murine leukemia virus (MLV) core and contain a luciferase reporter gene. As examples, the method to produce pseudotyped particles with the spike proteins of the highly pathogenic severe acute respiratory syndrome (SARS) and Middle East respiratory syndrome (MERS) coronaviruses are presented. The protocol describes the transfection procedure involved, how to infect susceptible target cells, and infectivity quantification by luciferase assay.

Since the entry steps of the pseudovirions are governed by the coronavirus $S$ at their surface, they enter cells in a similar fashion to native counterparts. As such, they are excellent surrogates of functional infectivity assays. Pseudotyped particles are usually derived from parental model viruses such as retroviruses $\left(\mathrm{MLV}^{7,8,9,10,11,12,13,14,15,16,17,18,19,20,21,22}\right.$ and the lentivirus human immunodeficiency virus $\mathrm{HIV}^{23,24,25,26,27,28,29,30,31,32,33,34,35}$ ) or rhabdoviruses (vesicular stomatitis virus - VSV $\mathrm{VS}^{36,39,40,41,42,43,44,45,46,47}$ ). When used in pseudotyping, the parental viruses' genomes are modified to remove essential genes, rendering them defective for accomplishing a complete replication cycle. This feature allows them to be used in intermediate biosafety level facilities (BSL-2) and is an important advantage over using highly pathogenic native viruses that require higher biosafety facilities (BSL-3, BSL-4 which are not as readily available) when conducting virus entry studies. 
Here, the $S$ proteins of risk group 3 pathogens SARS-CoV and MERS-CoV are used as examples of viral envelope proteins being incorporated into MLV pseudotyped particles, generating SARS-CoV S and MERS-CoV S pseudovirions (SARS-Spp and MERS-Spp, respectively). These pseudovirions have been successfully used in studies focusing on entry events of these viruses ${ }^{48,49,50,51}$. Another advantage is that the technique described here is not limited to pseudotyping coronavirus $S$ proteins: it is very flexible and can be used to incorporate representatives of all three classes of viral fusion proteins. Examples include influenza hemagglutinin (HA, class I) ${ }^{52}$, Ebola virus glycoprotein (GP class I), E1 protein of the alphavirus Semliki Forest virus (SFV, class II), and VSV glycoprotein (G, class III) ${ }^{53}$. In addition, more than one kind of viral glycoprotein can be co-incorporated into a pseudotyped particle, as in the case of influenza HA- and NA- pseudotyped particles ${ }^{51}$.

Based on the work performed by Bartosch et al. ${ }^{20}$, this protocol describes the generation of MLV pseudotyped particles with a three-plasmid co-transfection strategy using the widely available and highly transfection-competent HEK-293T cell line ${ }^{54}$. The first plasmid encodes the MLV core genes gag and $\mathrm{pol}$ but lacks the MLV envelope env gene. The second plasmid is a transfer vector that encodes a firefly luciferase reporter gene, an MLV $\Psi$-RNA packaging signal, along with 5'- and 3'-flanking MLV long terminal repeat (LTR) regions. The third plasmid encodes the fusion protein of interest, in this case either the SARS-CoV S or MERS-CoV S protein. Upon co-transfection of the three plasmids using a transfection reagent, viral RNA and proteins get expressed within transfected cells allowing generation of pseudotyped particles. Since MLV is used as pseudovirion backbone, this occurs at the plasma membrane: the RNAs containing the luciferase gene reporter and packaging signal get encapsulated into nascent particles that also incorporate plasma membrane-expressed coronavirus spike proteins. The particles that bud out from cells contain the coronavirus $S$ protein at their surface and are harvested for use in infectivity assays. Because pseudotyped particles harbor the coronavirus S protein and not the MLV envelope protein, when used for infecting cells, they behave like their native coronavirus counterparts for entry steps. The viral RNA containing the luciferase reporter and flanking LTRs is then released within the cell and the retroviral polymerase activities enable its reverse transcription into DNA and integration into the host cell genome. Quantification of the infectivity of viral pseudotyped particles in infected cells is then performed with a simple luciferase activity assay. Because the DNA sequence that gets integrated into the host cell genome only contains the luciferase gene and none of the MLV or coronavirus protein-encoding genes, they are inherently safer to use than replication-competent native viruses.

In addition to being safer surrogates and highly adaptable to allow incorporation of various kinds of envelope glycoproteins, the pseudotyped particles described here are also highly versatile and can be used to study many aspects of virus entry. This includes but is not limited to: testing host cell susceptibility to virus infection, analyzing the cellular entry pathways an enveloped virus uses, studying the effects of pharmacological inhibitors and drug screenings, conducting neutralization antibody assays, characterizing host cell entry of enveloped viruses that cannot be cultured, and generating viral vectors for gene delivery, stable cellular expression of genes of interest, or gene silencing.

\title{
1. Cell Seeding for Pseudotyped Particle Production
}

\author{
NOTE: Perform this step in the biosafety cabinet.
}

1. By standard cell culture techniques, obtain an $80-90 \%$ confluent $75 \mathrm{~cm}^{2}$ flask of HEK-293T/17 cells passaged in complete Dulbecco's Modified Eagle's Medium (DMEM-C) containing 10\% (vol/vol) fetal bovine serum (FBS), 10 mM 4-(2-hydroxyethyl)-1piperazineethanesulfonic acid (HEPES), $100 \mathrm{IU} / \mathrm{mL}$ penicillin, and $100 \mu \mathrm{g} / \mathrm{mL}$ streptomycin. Prepare DMEM-T medium for transfections (its composition is the same as DMEM-C but without antibiotics).

2. Wash cells with $10 \mathrm{~mL}$ of pre-warmed $\left(37^{\circ} \mathrm{C}\right)$ Dulbecco's Phosphate Buffered Saline (DPBS) twice. NOTE: Handle HEK293T/17 cells with care as they easily detach.

3. Aspirate the supernatant and detach cells with $1 \mathrm{~mL}$ of $0.25 \%$ trypsin solution pre-warmed at $37^{\circ} \mathrm{C}$. Place the flask of cells at $37{ }^{\circ} \mathrm{C}, 5 \%$ CO incubator for 3-5 min or until cells start detaching. NOTE: Avoid incubating cells with trypsin for more than $5 \mathrm{~min}$ as this typically leads to cell clumping.

4. Deactivate trypsin by adding $4 \mathrm{~mL}$ of DMEM-C medium and count cells using a cell counting slide and light microscope. NOTE: To avoid having to count too many cells, an additional dilution step may be required beforehand. Remember to factor in this dilution when calculating the actual cell density of trypsinized cells.

5. Dilute cells to $5 \times 10^{5}$ cells $/ \mathrm{mL}$ with DMEM-C.

6. Seed 6-well tissue culture plate with $2 \mathrm{~mL}$ of cell solution per well and gently move the plate back and forth and side to side to evenly distribute cells, avoiding circular motion.

NOTE: This is a key step. Evenly distributed cells will ensure that cells do not clump at the center of wells. In turn, this will ensure good transfection efficiencies and pseudotyped particle production.

7. Incubate the plate overnight $(16-18 \mathrm{~h})$ in a $37^{\circ} \mathrm{C}, 5 \% \mathrm{CO}_{2}$ cell culture incubator.

\section{Three-plasmid Co-transfection}

NOTE: Perform this step in the biosafety cabinet.

1. Observe cells under an inverted light microscope to check for the cell morphology and density.

NOTE: Ideally, cell density should be in the $40-60 \%$ confluency range. It is critical that cells are neither too confluent ( $80-90 \%$ confluent) nor too sparsely distributed (20-30\% confluent) in each well. A cell density of $40-60 \%$ confluency will ensure good pseudotyped particles production.

2. Plasmids mix

1. Calculate the plasmid mix for each envelope glycoprotein following the quantities for one well of a 6-well plate shown in Table 1. Multiply quantities if transfecting several wells and include an extra well to avoid running out of mix.

NOTE: Along with the SARS-CoV S and MERS-CoV S encoding plasmids, include empty vector control for the generation of negative control particles which lack viral envelope glycoproteins ( $\Delta$ env particles) along with a positive control glycoprotein such as vesicular 
stomatitis virus (VSV) G glycoprotein that is known to robustly infect a very wide range of cells (VSV-Gpp). Plasmids are available upon request.

2. Mix calculated volumes of plasmids and reduced serum cell culture medium (see Table of Materials) in a microcentrifuge tube.

3. Lipid-based transfection reagent mix (see Table of Materials)

1. Calculate the volumes for the transfection reagent mix from the quantities shown in Table 2 for one well (1:3 transfection ratio, multiply quantities as needed). Include extra wells to avoid running out of transfection reagent mix.

2. Mix calculated volumes of lipid-based transfection reagent ( $3 \mu \mathrm{L}$ per well) and reduced serum cell culture medium $(47 \mu \mathrm{L}$ per well) in a microcentrifuge tube, making sure to add the transfection reagent into the reduced serum cell culture medium and not the other way around.

4. Incubate both mixes (for one well: $50 \mu \mathrm{L}$ of plasmids mix and $50 \mu \mathrm{L}$ of lipid-based transfection reagent mix) separately for 5 min at room temperature.

5. Add the contents of the transfection reagent mix to the plasmids mix at a 1:1 ratio (for 1 well: $50 \mu \mathrm{L}$ of each mix)

6. Perform thorough up-down pipetting of the resulting mix.

7. Incubate the mix for at least $20 \mathrm{~min}$ at room temperature.

8. Aspirate the spent medium of cells.

9. Add gently $1 \mathrm{~mL}$ of pre-warmed $\left(37^{\circ} \mathrm{C}\right)$ reduced serum cell culture medium per well.

10. Add dropwise $100 \mu \mathrm{L}$ of transfection mix to each well.

NOTE: Exercise care when adding the transfection mix to wells of HEK-293T as they detach easily.

11. Incubate cells in a $37^{\circ} \mathrm{C}, 5 \% \mathrm{CO}_{2}$ cell culture incubator for $4-6 \mathrm{~h}$.

12. Add $1 \mathrm{~mL}$ per well of pre-warmed $\left(37^{\circ} \mathrm{C}\right)$ DMEM-T medium, which does not contain antibiotics

NOTE: This is a key step. Transfection reagents increase cell permeability and increase sensitivity to antibiotics. To ensure good transfection efficiency and pseudotyped particle production, it is important to avoid using cell culture medium containing antibiotics

13. Incubate cells in a $37^{\circ} \mathrm{C}, 5 \% \mathrm{CO}_{2}$ cell culture incubator for $48 \mathrm{~h}$.

\section{Pseudotyped Particles Collection}

NOTE: Perform this step in the biosafety cabinet.

1. Observe cells under inverted light microscope to check for cell morphology and general condition. Also check the color of the medium which should be light pink/slightly orange.

NOTE: This is an important step. If there is too much cell death associated with the transfection or the medium color turned orange/yellow (acidic $\mathrm{pH}$ ), this will typically be associated with lower yields in infectious pseudotyped particles

2. Transfer supernatants of transfected cells to $50 \mathrm{~mL}$ conical centrifuge tubes.

3. Centrifuge tubes at $290 \times g$ for $7 \mathrm{~min}$ to remove cell debris.

4. Filter clarified supernatants through a sterile $0.45 \mu \mathrm{m}$ pore-sized filter.

5. Make small volume aliquots (e.g., $500 \mu \mathrm{L}$ or $1 \mathrm{~mL}$ ) of pseudotyped virus solution in cryovials.

6. Store at $-80^{\circ} \mathrm{C}$

NOTE: The protocol can be paused here. Pseudotyped particles are stable at $-80^{\circ} \mathrm{C}$ for many months but once thawed, avoid re-freezing them as they will lose infectivity

\section{Pseudotyped Particle Infection of Susceptible Cells}

NOTE: Perform this step in the biosafety cabinet.

1. Cell seeding of susceptible cells in 24 -well plate

1. Obtain by standard cell culture techniques $80-90 \%$ confluent $75 \mathrm{~cm}^{2}$ flask of susceptible cells: Vero-E6 cells for SARS-CoV pseudotyped particles (SARS-Spp) and Huh-7 cells for MERS-CoV S pseudotyped particles (MERS-Spp).

NOTE: To confirm whether the pseudotyped particles that have been produced are infectious, it is important to carefully choose an appropriate susceptible cell line for pseudovirion infectivity assays. Using poorly permissive cells will lead to low infectivity.

2. Wash cells twice with $10 \mathrm{~mL}$ of pre-warmed $\left(37^{\circ} \mathrm{C}\right)$ DPBS.

3. Aspirate the supernatant and detach cells with $1 \mathrm{~mL}$ of $0.25 \%$ trypsin solution pre-warmed at $37^{\circ} \mathrm{C}$. Place the flask of cells at $37{ }^{\circ} \mathrm{C}$, $5 \% \mathrm{CO}_{2}$ incubator for $3-5 \mathrm{~min}$ or until cells start detaching.

NOTE: Avoid incubating cells with trypsin for more than 5 minutes as this typically leads to cell clumping. Huh- 7 cells are especially sensitive to this effect.

4. Deactivate trypsin by adding DMEM-C medium and count cells using a cell counting slide and light microscope.

5. Dilute cells to $5 \times 10^{5}$ cells $/ \mathrm{mL}$ with DMEM-C.

6. Seed wells of a 24 -well plate with $0.5 \mathrm{~mL}$ of cell solution per well and gently move the plate back and forth and side to side to evenly distribute cells, avoiding circular motion

NOTE: This is a key step. Evenly distributed cells will ensure that cells do not clump at the center of wells. In turn, this will ensure good infectivity assays. For each pseudotyped particle (SARS-Spp, MERS-Spp) and condition, prepare three wells for three experimental replicates. Include wells for the non-infected (N.I.), empty vector $\Delta$ env particles and positive control particles such as VSV-Gpp

7. Incubate the plate overnight $(16-18 \mathrm{~h})$ in a $37^{\circ} \mathrm{C}, 5 \% \mathrm{CO}_{2}$ cell culture incubator

2. Pseudotyped particle infection

1. Observe cells under light microscope and visually confirm that there is a confluent carpet of cells.

2. Bring cryovials of pseudotyped virus to thaw on ice.

3. Wash cells three times with $0.5 \mathrm{~mL}$ pre-warmed $\left(37^{\circ} \mathrm{C}\right)$ DPBS 
NOTE: This is a key step. Cells that are not properly rinsed prior to infection typically lead to poor infectivity readouts

4. Aspirate the supernatants of cells.

5. Inoculate cells with $200 \mu \mathrm{L}$ of thawed pseudotyped particle solution.

6. Incubate cells in a $37^{\circ} \mathrm{C}, 5 \% \mathrm{CO}_{2}$ cell culture incubator for $1-2 \mathrm{~h}$.

7. Add $300 \mu \mathrm{L}$ of pre-warmed $\left(37^{\circ} \mathrm{C}\right)$ DMEM-C medium.

8. Incubate cells in a $37^{\circ} \mathrm{C}, 5 \% \mathrm{CO}_{2}$ cell culture incubator for $72 \mathrm{~h}$.

\section{Infectivity Quantification by Luciferase Assay Readout}

NOTE: Perform initial steps in the biosafety cabinet.

1. Thaw luciferin substrate (stored at $-80^{\circ} \mathrm{C}$ ) and $5 x$ luciferase assay lysis buffer (stored at $-20^{\circ} \mathrm{C}$ ) until they reach room temperature.

2. Dilute luciferase assay lysis buffer to $1 \mathrm{x}$ with sterile water.

3. Aspirate supernatants of cells infected with pseudotyped particles.

4. Add $100 \mu \mathrm{L}$ of $1 \mathrm{x}$ luciferase assay lysis buffer to each well.

5. Place the plate on a rocker and incubate for $15 \mathrm{~min}$ with rocking at room temperature (from this point onwards the plate can be handled outside of a biosafety cabinet).

6. Prepare microcentrifuge tubes for each well by adding $20 \mu \mathrm{L}$ of luciferin substrate in each tube.

7. Turn on the luminometer.

8. Perform luciferase activity measurement one well at a time by transferring $10 \mu \mathrm{L}$ of lysate to one tube containing $20 \mu \mathrm{L}$ of luciferin substrate.

9. Flick the tube gently to mix contents, but avoid displacing the liquid on walls of tube.

10. Place the tube in device and close lid.

11. Measure the luminescence value of the tube by using the luminometer.

12. Record the relative light unit's measurement.

13. Repeat steps 5.8-5.12 until all wells are analyzed.

NOTE: With the appropriate equipment such as a plate reading luminometer, this process can be performed automatically. The assay will need to be scaled to the plate format (e.g., 96-well plate format).

\section{Data Analysis}

1. Calculation and plotting of relative luciferase units' averages and standard deviations

1. Use a graph plotting software to calculate luciferase assay measurement averages and standard deviations of experimental and biological replicates.

2. Plot data as bar chart with standard deviations.

NOTE: When performing statistical analyses on data, make sure to include at least three biological replicates in data sets.

\section{Representative Results}

Representative results of infectivity assays of SARS-CoV S and MERS-CoV S pseudotyped particles are shown in Figure 1. As expected, for both Figure 1A and 1B, the VSV G pseudotyped positive control particles (VSV-Gpp) gave very high average infectivity in the $10^{6}$ to $10^{7}$ relative luciferase units (RLU) range respectively. For SARS-CoV S pseudotyped particles (Figure 1A) infection of susceptible Vero-E6 cells, a strong average infectivity was measured at around $9.8 \times 10^{4} \mathrm{RLU}$. This value is almost 3 orders of magnitude higher than the values measured for the non-infected control $\left(1.1 \times 10^{2} \mathrm{RLU}\right)$, or the $\Delta$ env particles $\left(1.5 \times 10^{2} \mathrm{RLU}\right)$, which do not harbor any viral envelope glycoproteins at their surface. Similarly, for MERS-CoV S pseudotyped particles (Figure 1B) infection of Huh-7 cells, a high average infectivity was measured at around 1.0 $\times 10^{6} \mathrm{RLU}$. This is almost 4 orders of magnitude higher than the values measured for the non-infected control $\left(0.8 \times 10^{2} \mathrm{RLU}\right)$, or the $\triangle \mathrm{env}$ particles $\left(2.0 \times 10^{2} \mathrm{RLU}\right)$. An additional infectivity assay was performed in which the SARS-Spp and MERS-Spp were serially diluted and used to infect Vero-E6 cells (Figure 2A). This assay confirms that the luciferase activity measured is dependent on the concentration of the particles used to infect cells. To confirm the role of angiotensin converting enzyme 2 (ACE2) and dipeptidyl peptidase 4 (DPP4), the receptors of SARSCoV and MERS-CoV, respectively, in mediating attachment and entry of the pseudotyped particles, we used poorly-permissive HEK-293T cells and transfected them to express either ACE2 or DPP4 (Figure 2B). The transfected cells were then used for an infectivity assay. This analysis demonstrates that upon overexpression of ACE2 and DPP4, there is a $\sim 4-\log$ and $\sim 2-\log$ increase for SARS-Spp and MERS-Spp infectivity, respectively, confirming that receptor usage of pseudovirions is similar to that of native viruses. 
The examples shown here demonstrate the importance of including negative (non-infected, $\Delta$ env particles) as well as positive control (VSV$\mathrm{Gpp}$ ) conditions when producing pseudotyped particles. Indeed, the positive control VSV-Gpp particles allow us to assess whether a particular batch of pseudotyped particles was successful in yielding functional and infective pseudovirions. Expected results of typical infection by VSVGpp particles in most mammalian cell lines are in the $10^{6}-10^{7} \mathrm{RLU}$ range. Problems with HEK-293T/17 producer cells (high passage number, issues with cell densities) or poor transfection efficiencies can impact overall pseudotyped particle production and infectivity. Furthermore, the negative control conditions are also important as they allow us to assess the baselines of RLU measurements in a particular cell line (noninfected condition) and non-specific internalization of particles ( $\Delta$ env infection) which is not mediated by a viral envelope protein. Ideally, for a given type of particle pseudotyped with a viral envelope protein of interest, it is recommended to obtain values that are a few orders of magnitude higher than the negative control values, as shown here in Figure 1A,B. However, if for a given cell type a pseudotyped virus infection gives very little infectivity (i.e., close to negative controls such as in Figure 2B in non-transfected N.T. conditions) it does not necessarily mean that the pseudotyped particle production was faulty. It could well be that the particular cell line used is not or poorly permissive to infection. It is recommended to check whether a given cell type is expected to be susceptible to infection by the virus being investigated. Transfecting poorly permissive cells with plasmid(s) expressing the viral receptor(s) can allow more efficient viral entry and infection to occur, as shown in Figure 2B, where upon transfection of ACE2 and DPP4 receptors in target HEK-293T cells, there is a $\sim 4-$ and $\sim 2-l o g$ increase in infectivity, respectively.

\begin{tabular}{|l|l|}
\hline Plasmid/reagent & Quantity \\
\hline pCMV-MLVgagpol MLV gag and pol encoding plasmid & $300 \mathrm{ng}$ \\
\hline pTG-Luc transfer vector with luciferase reporter & $400 \mathrm{ng}$ \\
\hline pcDNA-SARS-S, pcDNA-MERS-S or empty vector & $300 \mathrm{ng}$ \\
\hline Reduced serum cell culture medium & To $50 \mu \mathrm{L}$ \\
\hline
\end{tabular}

Table 1: Quantities of plasmids and reduced serum cell culture medium required to transfect one well of a 6-well plate of HEK-293T/17 cells for pseudotyped particle production. From the concentration of plasmid preparations, calculate the required volume to reach the required amount of plasmid DNA. If more than one well is transfected with the same plasmids, multiply volumes by required number of wells to transfect, and include an extra well in calculations to avoid running out of mix in later steps. The total amount of DNA being transfected is $1 \mu \mathrm{g} /$ well. Plasmids are available upon request to authors.

\begin{tabular}{|l|l|}
\hline Reagent & Quantity \\
\hline Transfection reagent & $3 \mu \mathrm{L}$ \\
\hline Reduced serum cell culture medium & $47 \mu \mathrm{L}$ \\
\hline
\end{tabular}

Table 2: Quantities of transfection reagent and reduced serum cell culture medium required to transfect one well of a 6-well plate of HEK-293T/17 cells for pseudotyped particle production. Multiply volumes by required number of wells to transfect and include extra wells in calculations to avoid running out of mix in later steps. The transfection reagent: plasmid DNA ratio used is 3:1.

A

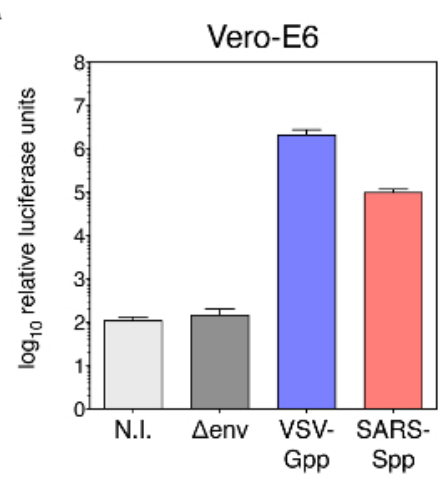

B

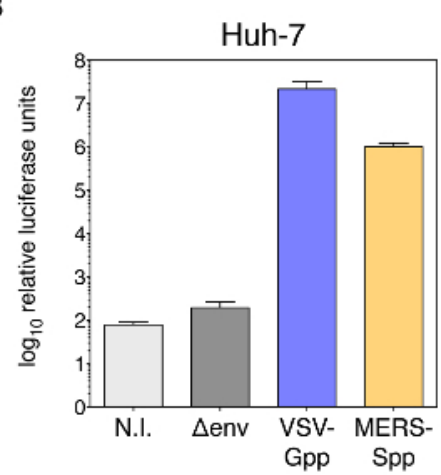

Figure 1: Coronavirus S-pseudotyped particle infectivity assays in susceptible host cells using a murine leukemia virus (MLV) backbone and luciferase reporter gene. (A) SARS-CoV S pseudotyped particle infectivity assay in Vero-E6 cells. (B) MERS-CoV S pseudotyped particle infectivity assay in Huh-7 cells. For both $(\mathbf{A})$ and $(\mathbf{B})$, plotted data corresponds to the average relative luciferase units from three independent experiments, with error bars corresponding to standard deviation (s.d.). Data plotted in log $\log _{10}$ scale on y-axis. N.I.: non-infected control; $\Delta$ env: infection with pseudotyped particles lacking viral envelope glycoproteins and VSV-Gpp: infection with pseudotyped particles bearing positive control VSV G envelope glycoprotein. Other abbreviation used, SARS-Spp: infection with SARS-CoV S pseudotyped particles, MERS-Spp: infection with MERS-CoV S pseudotyped particles. Please click here to view a larger version of this figure. 
A

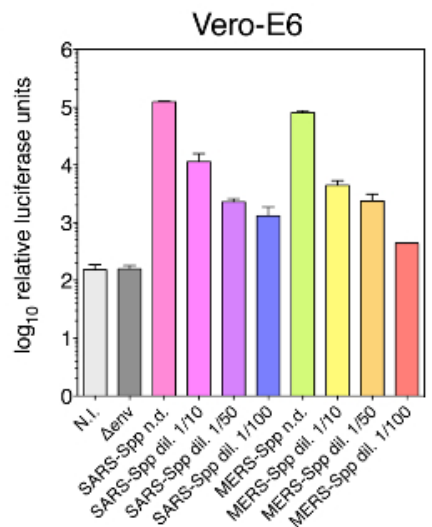

B

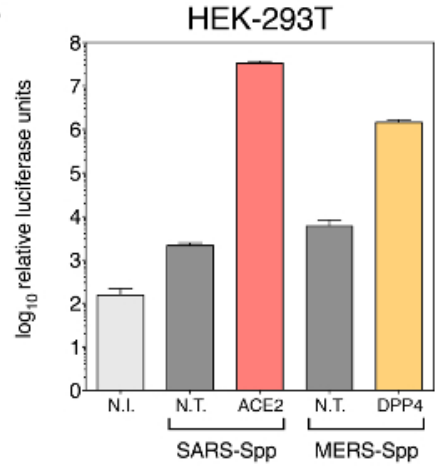

Figure 2: Concentration-dependence of CoV S-pseudovirion infectivity and role of ACE2 and DPP4 receptors in SARS-Spp and MERSSpp entry. (A) Concentration-dependence of SARS-Spp and MERS-Spp infectivity measured by luciferase activity assay. Pseudovirions were serially diluted and used to infect Vero-E6 cells. (B) Infectivity assay of SARS-Spp and MERS-Spp in poorly permissive HEK-293T target cells transfected to express ACE2, and DPP4 receptors, respectively. Data plotted in $\log _{10}$ scale on y-axis as in Figure 1 from duplicate experiments, with error bars corresponding to standard deviation (s.d.). N.T.: non-transfected control HEK-293T cells; ACE2: angiotensin converting enzyme 2 (SARS-CoV receptor) and DPP4: dipeptidyl peptidase 4 (MERS-CoV receptor). Other abbreviations used are the same as in Figure 1. Please click here to view a larger version of this figure.

\section{Discussion}

This protocol describes a method to efficiently produce pseudotyped particles bearing the S protein of risk group 3 coronaviruses, SARS-CoV and MERS-CoV, in a BSL-2 setting. These particles, which incorporate a luciferase reporter gene, enable us to easily quantify coronavirus Smediated entry events by a relatively simple luciferase assay ${ }^{48,49,50,51}$. In infectivity assays using permissive cells, we confirm that the luciferase activity measured is dependent on the concentration of particles. In addition, ACE2 and DPP4 receptor transfection allows for more efficient entry in poorly permissive cells lines, such as HEK-293T cells. The method is highly adaptable to other viral envelope glycoproteins and has been used extensively ${ }^{48,49,50,51,52,53,55,56,57,58,59}$, often to complement other assays like biochemical analyses or native virus infections.

The protocol we describe here is based on the retrovirus MLV that incorporates a luciferase reporter. However, it is important to emphasize that there is a very wide array of other pseudotyping systems that have been successfully developed for packaging coronavirus $S^{12,13,25,26,30,31,32}$ and other viral envelope glycoproteins ${ }^{10,11,14,16,17,23,24,29,33,38,40,42,44,46}$. Some of these other systems are based on the commonly used MLV retroviral core $7,8,9,10,11,12,13,14,15,16,17,18,19,20$, or based on the widely used lentiviral HIV-1 pseudotyping system using different strategies $^{23,24,25,26,27,28,29,30,31,32,33,34,35}$, or with the rhabdovirus vesicular stomatitis virus (VSV) as core, which allows to incorporate a wide variety of envelope glycoproteins, and again with various strategies employed $37,38,39,40,41,42,43,44,45,46,47$. In addition, other reporters such as fluorescent proteins like GFP ${ }^{11,13}$ and RFP ${ }^{36}$, or enzymes other than luciferase like $\beta$-galactosidase ${ }^{16,17}$ and secreted alkaline phosphatase $(\mathrm{SEAP})^{42}$ have been successfully employed for measurements. Furthermore, in the assay presented in this protocol, a transient transfection was used to express the MLV and CoV S genes and proteins. However, there are other strategies for expression, such as generation of stable cell lines for production of pseudotyped viruses ${ }^{7,14}$. As each of these systems have their advantages and disadvantages, it is important to consider the following important parameters when deciding which pseudotyping system best suits an investigator's needs: pseudovirion core (MLV, HIV-1, VSV or others), how selective a particular pseudotyping core is in incorporating a specific viral envelope glycoprotein, reporter for assay readout (GFP, luciferase, SEAP or other), and the transfection strategy (number of plasmids involved in co-transfection, transient transfection or generation of stable cell lines).

There are a number of critical steps in the method that are important to emphasize. Cell density, particularly of the HEK-293T/17 producer cell line is a critical factor in ensuring successful transfection. A cell density in the range of $40-60 \%$ confluency was found to be optimal. Higher densities typically result in low transfection efficiencies and low particle production. Also, it is important to keep in mind that HEK-293T/17 cells are less adherent than other cell lines. Care should be exercised when handling them to avoid detaching them unnecessarily. One option is to treat cell culture plastic surfaces with poly-D-lysine to enhance adherence. Furthermore, higher cell passage often results in poor transfection rates. After adding the transfection reagent to HEK-293T/17 cells, it is also important to remember that cell permeability increases. This is why at this point it is best to avoid using medium containing antibiotics as they may increase cytotoxicity. Before collecting pseudotyped particles, check the color of transfected HEK-293T/17 cell supernatants. Typically, after $48 \mathrm{~h}$ of transfection, the cell culture medium color takes an orange-pink tinge. Yellow-colored medium usually translates to poor pseudotyped particles yields and is often a result of issues with cell seeding density or high passage number.

In this protocol, pseudotyped particle production is performed in a 6-well plate format. To increase volume of produced particles, several wells of a 6 -well plate can be transfected with the same plasmids mix and the supernatants can be pooled together. The pool can then be clarified, filtered and aliquoted. Alternatively, to scale production up, other kinds of vessels (e.g., 25 , or $75 \mathrm{~cm}^{2}$ flasks) can be used. In this case, transfection conditions should be scaled up accordingly. In this protocol, the infectivity assay is performed using a 24-well plate format and a luminometer that only allows measurements one tube at a time. For high throughput screenings, other formats are also possible, such as 96-well plate format and a plate reader luminometer. Volumes and reagents for the luciferase assay need to be adapted accordingly. Storage of pseudotyped particles in cryovials at $-80^{\circ} \mathrm{C}$ maintains their stability for several months without noticeable decrease in infectivity. It is not recommended to subject them to freeze-thaw cycles as this will decrease their infectivity over time. Thus, it is best to store them in small aliquots such as $0.5-1 \mathrm{~mL}$ and thaw them before an infection. 
The method presented here has several limitations. An important one is the fact that pseudotyped particles recapitulate only viral entry events. To analyze other steps in the infectious life cycle, other assays are required. Furthermore, as MLV particles bud at the plasma membrane, it is important to bear in mind that the envelope glycoprotein being studied needs to also traffic to the plasma membrane for incorporation into pseudovirions during production. As such, it is important to know where in the cell a particular viral envelope glycoprotein is expressed in transfection conditions, such as by visualizing subcellular localization with an immunofluorescence assay, and/or by checking for retention signals within the protein. Also, while the protocol describes steps to produce and test infectivity, it does not detail how to measure incorporation of viral envelope glycoproteins into pseudotyped particles. One method is to perform western blot assays on concentrated solutions of particles, as previously described ${ }^{50,51}$ for MERS-CoV S incorporation. In these assays, the $S$ envelope glycoprotein of MERS-CoV is probed along with the capsid (p30) protein of MLV, which allow us to normalize incorporation of the S protein into particles. Other examples of such assays analyzing viral envelope glycoprotein incorporation into pseudovirions have been performed for SARS-CoV S incorporation in an HIV-1 lentiviral pseudovirion system ${ }^{32}$, Ebola glycoprotein (GP) in another MLV pseudotyped particle system ${ }^{17}$, and influenza hemagglutinin (HA) and neuraminidase (NA) in VSV pseudovirions ${ }^{38}$. A recent development in characterizing pseudotyped particle production is the use of innovative imaging devices such as Nanosight: it enables us to directly visualize, quantify, and size viral particles ${ }^{50}$. The device provides detailed information on overall particle production; however, it is important to keep in mind that it does not provide information on envelope glycoprotein incorporation. A future direction for the application of these versatile pseudovirion particles is to analyze individual viral fusion events using single particle tracking, microfluidics and total internal reflection fluorescence microscopy ${ }^{60,61,62}$. Such approaches were successfully applied to influenza virus and feline coronavirus particles as well as influenza HA- and NA-pseudotyped VSV-based pseudovirions ${ }^{63}$. The deployment of such techniques applied to coronavirus S-pseudotyped MLV-based particles is currently being developed.

\section{Disclosures}

The authors have nothing to disclose.

\section{Acknowledgments}

We wish to thank all members of the Whittaker and Daniel labs for helpful comments. Research funding was provided by the NIH grants R21 Al111085 and R01 Al135270. T.T. acknowledges support from the Presidential Life Science Fellowship in Cornell University and National Science Foundation Graduate Research Fellowship Program under Grant No. DGE-1650441. L.N. acknowledges support from a Samuel C

Fleming Family Graduate Fellowship and National Science Foundation Graduate Research Fellowship Program under Grant No. DGE-1650441. This work is also supported by National Science Foundation 1504846 (to S.D. and G.R.W.).

\section{References}

1. Dimitrov, D. S. Virus entry: molecular mechanisms and biomedical applications. Nature Reviews Microbiology. 2 (2), 109-122 (2004).

2. White, J. M., Delos, S. E., Brecher, M., Schornberg, K. Structures and Mechanisms of Viral Membrane Fusion Proteins: Multiple Variations on a Common Theme. Critical Reviews in Biochemistry and Molecular Biology. 43 (3), 189-219 (2008).

3. Bosch, B. J., van der Zee, R., de Haan, C. A. M., Rottier, P. J. M. The Coronavirus Spike Protein Is a Class I Virus Fusion Protein: Structural and Functional Characterization of the Fusion Core Complex. Journal of Virology. 77 (16), 8801-8811 (2003).

4. Belouzard, S., Millet, J. K., Licitra, B. N., Whittaker, G. R. Mechanisms of Coronavirus Cell Entry Mediated by the Viral Spike Protein. Viruses. 4 (6), 1011-1033 (2012).

5. Millet, J. K., Whittaker, G. R. Physiological and molecular triggers for SARS-CoV membrane fusion and entry into host cells. Virology. 517 3-8 (2018).

6. Millet, J. K., Whittaker, G. R. Host cell proteases: Critical determinants of coronavirus tropism and pathogenesis. Virus Research. 202 (0), 120-134 (2015).

7. Steidl, S. et al. Coreceptor Switch of [MLV(SIVagm)] Pseudotype Vectors by V3-Loop Exchange. Virology. 300 (2), 205-216 (2002).

8. Höhne, M., Thaler, S., Dudda, J. C., Groner, B., Schnierle, B. S. Truncation of the Human Immunodeficiency Virus-Type-2 Envelope Glycoprotein Allows Efficient Pseudotyping of Murine Leukemia Virus Retroviral Vector Particles. Virology. 261 (1), $70-78$ (1999).

9. Wang, W. et al. Establishment of retroviral pseudotypes with influenza hemagglutinins from $\mathrm{H} 1, \mathrm{H} 3$, and $\mathrm{H} 5$ subtypes for sensitive and specific detection of neutralizing antibodies. Journal of Virological Methods. 153 (2), 111-119 (2008).

10. Wallerstrom, S. et al. Detection of antibodies against $\mathrm{H} 5$ and $\mathrm{H} 7$ strains in birds: evaluation of influenza pseudovirus particle neutralization tests. Infection Ecology, Epidemiology. 4 (2014).

11. Radoshitzky, S. R. et al. Transferrin receptor 1 is a cellular receptor for New World haemorrhagic fever arenaviruses. Nature. 446 (7131), 92-96 (2007).

12. Han, D. P., Kim, H. G., Kim, Y. B., Poon, L. L., Cho, M. W. Development of a safe neutralization assay for SARS-CoV and characterization of S-glycoprotein. Virology. 326 (1), 140-149 (2004).

13. Moore, M. J. et al. Retroviruses pseudotyped with the severe acute respiratory syndrome coronavirus spike protein efficiently infect cells expressing angiotensin-converting enzyme 2. Journal of Virology. 78 (19), 10628-10635 (2004).

14. Sharkey, C. M., North, C. L., Kuhn, R. J., Sanders, D. A. Ross River virus glycoprotein-pseudotyped retroviruses and stable cell lines for their production. Journal of Virology. 75 (6), 2653-2659 (2001).

15. Bruett, L., Clements, J. E. Functional murine leukemia virus vectors pseudotyped with the visna virus envelope show expanded visna virus cell tropism. Journal of Virology. 75 (23), 11464-11473 (2001).

16. Ma, M. et al. Murine leukemia virus pseudotypes of La Crosse and Hantaan Bunyaviruses: a system for analysis of cell tropism. Virus Research. 64 (1), 23-32 (1999).

17. Wool-Lewis, R. J., Bates, P. Characterization of Ebola virus entry by using pseudotyped viruses: identification of receptor-deficient cell lines. Journal of Virology. 72 (4), 3155-3160 (1998).

18. Op De Beeck, A. et al. Characterization of Functional Hepatitis C Virus Envelope Glycoproteins. Journal of Virology. 78 (6), $2994-3002$ (2004). 
19. Calland, N. et al. (-)-Epigallocatechin-3-gallate is a new inhibitor of hepatitis C virus entry. Hepatology. 55 (3), $720-729$ (2012).

20. Bartosch, B., Dubuisson, J., Cosset, F. L. Infectious hepatitis C virus pseudo-particles containing functional E1-E2 envelope protein complexes. Journal of Experimental Medicine. 197 (5), 633-642 (2003).

21. Giroglou, T. et al. Retroviral vectors pseudotyped with severe acute respiratory syndrome coronavirus S protein. Journal of Virology. 78 (17), 9007-9015 (2004).

22. Dye, C., Temperton, N., Siddell, S. G. Type I feline coronavirus spike glycoprotein fails to recognize aminopeptidase $\mathrm{N}$ as a functional receptor on feline cell lines. Journal of General Virology. 88 (6), 1753-1760 (2007).

23. Kobinger, G. P., Weiner, D. J., Yu, Q.-C., Wilson, J. M. Filovirus-pseudotyped lentiviral vector can efficiently and stably transduce airway epithelia in vivo. Nature Biotechnology. 19225 (2001).

24. Salvador, B., Zhou, Y., Michault, A., Muench, M. O., Simmons, G. Characterization of Chikungunya pseudotyped viruses: Identification of refractory cell lines and demonstration of cellular tropism differences mediated by mutations in E1 glycoprotein. Virology. 393 (1), 33-41 (2009).

25. Nie, Y. et al. Highly infectious SARS-CoV pseudotyped virus reveals the cell tropism and its correlation with receptor expression. Biochemical and Biophysical Research Communications. 321 (4), 994-1000 (2004).

26. Grehan, K., Ferrara, F., Temperton, N. An optimised method for the production of MERS-CoV spike expressing viral pseudotypes. MethodsX. 2 379-384 (2015).

27. Bakri, Y. et al. The Maturation of Dendritic Cells Results in Postintegration Inhibition of HIV-1 Replication. The Journal of Immunology. 166 (6), 3780-3788 (2001)

28. Hsu, M. et al. Hepatitis $\mathrm{C}$ virus glycoproteins mediate $\mathrm{pH}$-dependent cell entry of pseudotyped retroviral particles. Proceedings of the National Academy of Sciences. 100 (12), 7271-7276 (2003).

29. Simmons, G. et al. DC-SIGN and DC-SIGNR Bind Ebola Glycoproteins and Enhance Infection of Macrophages and Endothelial Cells. Virology. 305 (1), 115-123 (2003).

30. Simmons, G. et al. Inhibitors of cathepsin L prevent severe acute respiratory syndrome coronavirus entry. Proceedings of the National Academy of Sciences. 102 (33), 11876-11881 (2005).

31. Gierer, S. et al. The spike-protein of the emerging betacoronavirus EMC uses a novel coronavirus receptor for entry, can be activated by TMPRSS2 and is targeted by neutralizing antibodies. Journal of Virology. 87 (10), 5502-5511 (2013).

32. Bertram, S. et al. Cleavage and activation of the SARS-coronavirus spike-protein by human airway trypsin-like protease. Journal of Virology. 85 (24), 13363-13372 (2011).

33. Bertram, S. et al. TMPRSS2 and TMPRSS4 facilitate trypsin-independent spread of influenza virus in Caco-2 cells. Journal of Virology. 84 (19), 10016-10025 (2010).

34. Connor, R. I., Chen, B. K., Choe, S., Landau, N. R. Vpr Is Required for Efficient Replication of Human Immunodeficiency Virus Type-1 in Mononuclear Phagocytes. Virology. 206 (2), 935-944 (1995).

35. Evans, M. J. et al. Claudin-1 is a hepatitis C virus co-receptor required for a late step in entry. Nature. 446 (7137), 801-805 (2007).

36. Negrete, O. A. et al. EphrinB2 is the entry receptor for Nipah virus, an emergent deadly paramyxovirus. Nature. 436 (7049), 401-405 (2005).

37. Suda, Y. et al. Analysis of the entry mechanism of Crimean-Congo hemorrhagic fever virus, using a vesicular stomatitis virus pseudotyping system. Archives of Virology. 161 (6), 1447-1454 (2016).

38. Zimmer, G., Locher, S., Berger Rentsch, M., Halbherr, S. J. Pseudotyping of vesicular stomatitis virus with the envelope glycoproteins of highly pathogenic avian influenza viruses. Journal of General Virology. 95 (Pt 8), 1634-1639 (2014).

39. Moeschler, S., Locher, S., Conzelmann, K. K., Kramer, B., Zimmer, G. Quantification of Lyssavirus-Neutralizing Antibodies Using Vesicular Stomatitis Virus Pseudotype Particles. Viruses. 8 (9) (2016).

40. Tong, W., Yin, X. X., Lee, B. J., Li, Y. G. Preparation of vesicular stomatitis virus pseudotype with Chikungunya virus envelope protein. Acta Virologica. 59 (02), 189-193 (2015).

41. Tani, H. et al. Involvement of ceramide in the propagation of Japanese encephalitis virus. Journal of Virology. 84 (6), $2798-2807$ (2010).

42. Kaku, Y. et al. Second generation of pseudotype-based serum neutralization assay for Nipah virus antibodies: sensitive and high-throughput analysis utilizing secreted alkaline phosphatase. Journal of Virological Methods. 179 (1), 226-232 (2012).

43. Tani, H. et al. Analysis of Lujo virus cell entry using pseudotype vesicular stomatitis virus. Journal of Virology. 88 (13), 7317-7330 (2014).

44. Ogino, M. et al. Use of Vesicular Stomatitis Virus Pseudotypes Bearing Hantaan or Seoul Virus Envelope Proteins in a Rapid and Safe Neutralization Test. Clinical and Vaccine Immunology. 10 (1), 154-160 (2003).

45. Logan, N. et al. Efficient generation of vesicular stomatitis virus (VSV)-pseudotypes bearing morbilliviral glycoproteins and their use in quantifying virus neutralising antibodies. Vaccine. 34 (6), 814-822 (2016).

46. Takada, A. et al. A system for functional analysis of Ebola virus glycoprotein. Proceedings of the National Academy of Sciences. 94 (26), 14764-14769 (1997).

47. Whitt, M. A. Generation of VSV pseudotypes using recombinant DeltaG-VSV for studies on virus entry, identification of entry inhibitors, and immune responses to vaccines. Journal of Virological Methods. 169 (2), 365-374 (2010).

48. Lai, A. L., Millet, J. K., Daniel, S., Freed, J. H., Whittaker, G. R. The SARS-CoV Fusion Peptide Forms an Extended Bipartite Fusion Platform that Perturbs Membrane Order in a Calcium-Dependent Manner. Journal of Molecular Biology. 429 (24), 3875-3892 (2017).

49. Millet, J. K. et al. Middle East respiratory syndrome coronavirus infection is inhibited by griffithsin. Antiviral Research. 133 1-8 (2016).

50. Millet, J. K. et al. A camel-derived MERS-CoV with a variant spike protein cleavage site and distinct fusion activation properties. Emerging Microbes, Infections. 5 (12), e126 (2016).

51. Millet, J. K., Whittaker, G. R. Host cell entry of Middle East respiratory syndrome coronavirus after two-step, furin-mediated activation of the spike protein. Proceedings of the National Academy of Sciences. 111 (42), 15214-15219 (2014).

52. Tse, L. V., Hamilton, A. M., Friling, T., Whittaker, G. R. A Novel Activation Mechanism of Avian Influenza Virus H9N2 by Furin. Journal of Virology. 88 (3), 1673-1683 (2014).

53. Sun, X., Belouzard, S., Whittaker, G. R. Molecular architecture of the bipartite fusion loops of vesicular stomatitis virus glycoprotein G, a class III viral fusion protein. Journal of Biological Chemistry. 283 (10), 6418-6427 (2008).

54. Millet, J., Whittaker, G. Murine Leukemia Virus (MLV)-based Coronavirus Spike-pseudotyped Particle Production and Infection. Bio-Protocol. 6 (23), e2035 (2016).

55. Bonnin, A., Danneels, A., Dubuisson, J., Goffard, A., Belouzard, S. HCoV-229E spike protein fusion activation by trypsin-like serine proteases is mediated by proteolytic processing in the S2' region. Journal of General Virology. 10.1099/jgv.0.001074 (2018). 
56. Belouzard, S., Madu, I., Whittaker, G. R. Elastase-mediated activation of the SARS coronavirus spike protein at discrete sites within the S2 domain. Journal of Biological Chemistry. 285 (30), 22758-22763 (2010).

57. Madu, I. G., Roth, S. L., Belouzard, S., Whittaker, G. R. Characterization of a Highly Conserved Domain within the Severe Acute Respiratory Syndrome Coronavirus Spike Protein S2 Domain with Characteristics of a Viral Fusion Peptide. Journal of Virology. 83 (15), $7411-7421$ (2009).

58. Madu, I. G., Belouzard, S., Whittaker, G. R. SARS-coronavirus spike S2 domain flanked by cysteine residues C822 and C833 is important for activation of membrane fusion. Virology. 393 (2), 265-271 (2009).

59. Belouzard, S., Chu, V. C., Whittaker, G. R. Activation of the SARS coronavirus spike protein via sequential proteolytic cleavage at two distinct sites. Proceedings of the National Academy of Sciences. 106 (14), 5871-5876 (2009).

60. Costello, D. A., Millet, J. K., Hsia, C.-Y., Whittaker, G. R., Daniel, S. Single particle assay of coronavirus membrane fusion with proteinaceous receptor-embedded supported bilayers. Biomaterials. 34 (32), 7895-7904 (2013).

61. Costello, D. A., Hsia, C.-Y., Millet, J. K., Porri, T., Daniel, S. Membrane Fusion-Competent Virus-Like Proteoliposomes and Proteinaceous Supported Bilayers Made Directly from Cell Plasma Membranes. Langmuir. 29 (21), 6409-6419 (2013).

62. Floyd, D. L., Ragains, J. R., Skehel, J. J., Harrison, S. C., van Oijen, A. M. Single particle kinetics of influenza virus membrane fusion. Proceedings of the National Academy of Sciences. 105 (40), 15382-15387 (2008).

63. Hsu, H.-L., Millet, J. K., Costello, D. A., Whittaker, G. R., Daniel, S. Viral fusion efficacy of specific H3N2 influenza virus reassortant combinations at single-particle level. Scientific reports. 635537 (2016). 